\title{
Microcirurgia para aneurismas intracranianos múltiplos: série de 29 casos
}

\author{
Pablo Rodrigues de Carvalho', Marcelo Nery Silva², José Carlos Rodrigues \\ Júnior ${ }^{3}$, Bernardo Alves Barbosa1 , Eduardo Monteiro de Oliveira1 ${ }^{1}$ Breno Ner ${ }^{1}$, \\ Bruna Nayana Ribeiro Barbosa ${ }^{1}$, Ricardo Marques Lopes de Araújo' \\ Hospital Heliópolis, Santo Antônio, São Caetano do Sul, SP, Brasil.
}

\section{RESUMO}

Objetivo: Apresentar 23 pacientes com 52 aneurismas tratados cirurgicamente em uma única cirurgia em um serviço de neurocirurgia da secretaria estadual de saúde de São Paulo, entre os anos de 2009 e 2011. Método: Análise retrospectiva dos prontuários de pacientes submetidos à clipagem de dois ou mais aneurismas cerebrais, em uma única cirurgia, no período de janeiro de 2007 a julho de 2012. Resultados: Vinte e nove pacientes foram submetidos à clipagem de dois ou mais aneurismas cerebrais em uma única cirurgia - 28 por meio de uma única craniotomia e um por meio de duas craniotomias. Desses, 20, 7, 1 e 1 foram submetidos, respectivamente, à clipagem de 2, 3, 4 e 5 aneurismas cerebrais. Cinco eram do sexo masculino e 24 do sexo feminino, e a faixa etária esteve entre 40 e 66 anos. Oito craniotomias à esquerda foram realizadas para abordagem de 17 aneurismas cerebrais lateralizados à esquerda e cinco à direita, assim como três aneurismas de complexo comunicante anterior. Doze craniotomias à direita foram realizadas para abordagem de 23 aneurismas cerebrais lateralizados à direita e seis à esquerda, assim como 15 aneurismas de complexo comunicante anterior e um de artéria basilar. Dos 29 pacientes, 28 evoluíram com 1-3 pts, e somente um evolui com 4-5 pts na escala de Rankin seis meses após a cirurgia. Conclusão: Preconizamos abordagem microneurocirúrgica para a maioria dos casos de aneurismas intracranianos múltiplos, buscando a clipagem microcirúrgica de todos os aneurismas intracranianos, se viável, em um único estágio e uma única craniotomia.

\section{PALAVRAS-CHAVE}

Microcirurgia, aneurisma intracraniano, craniotomia.

\section{ABSTRACT}

Microsurgery for multiple intracranial aneurysms: a review of 29 cases

Objective: Present 23 patients with 52 aneurysms treated surgically in a single surgery in a neurosurgical service of the state board of health of São Paulo between the years 2009 and 2011. Method: Retrospective analysis of patients undergoing clipping of two or more cerebral aneurysms in a single surgery, from January 2007 to July 2012. Results: Twenty-nine patients underwent two or more clipping cerebral aneurysms in a single operation - 28 with a single craniotomy and one through two craniotomies. Of these, 20, 7, 1 and 1 were submitted respectively to the clipping 2, 3, 4 and 5 cerebral aneurysms. Five were male and 24 were female, and the age range was between 40 and 66 years old. Eight left craniotomies were performed to approach 17 lateralized brain aneurysms to the left and five to the right, as well as three anterior communicating complex aneurysms. Twelve craniotomies was performed on the right to approach 23 intracranial aneurysms lateralized to the right and six on the left, as well as 15 aneurysms complex anterior communicating and 1 on the basilar artery. Of the 29 patients, 28 evolved with 1-3 pts and only one with 4-5 pts on the Rankin scale six months after surgery. Conclusion: We advocate microsurgical approach for most cases of multiple intracranial aneurysms aiming the microsurgical clipping of all intracranial aneurysms if feasible through a single stage and a single craniotomy.

\section{KEYWORDS}

Microsurgery, intracranial aneurysm, craniotomy.

1 Residente de Neurocirurgia do Hospital Heliópolis, São Caetano do Sul, SP, Brasil. 


\section{Introdução}

Aneurismas intracranianos múltiplos são identificados em 15\%-45\% dos pacientes com diagnóstico de hemorragia subaracnóidea ${ }^{1-3} \mathrm{e}$, comumente, implicam um manejo terapêutico complexo e uma morbimortalidade significativamente superior à dos casos de aneurisma intracraniano único. ${ }^{4-7}$

Inúmeros fatores de risco estão associados a incidência e prevalência, todavia a patogênese e, com efeito, os mecanismos implicados na formação de aneurismas intracranianos múltiplos permanecem indeterminados. ${ }^{1,2,8}$

O estudo diagnóstico busca, principalmente, determinar o sítio da ruptura, e/ou com alto risco de ruptura, e compreender a morfologia, localização e relações neurais e vasculares dos aneurismas intracranianos.

O manejo terapêutico é variável na seleção da modalidade (clipagem microcirúrgica, endovascular ou multimodal), do timing (precoce ou tardio) e dos estágios (em um estágio ou múltiplos estágios) de tratamento, não havendo consenso na literatura sobre o tema.

O tratamento cirúrgico permanece associado a uma alta frequência de complicações, apesar dos avanços nas técnicas cirúrgicas e anestésicas, todavia possibilita o tratamento definitivo de virtualmente todos os aneurismas intracranianos, frequentemente, por meio de uma craniotomia e em um único estágio. ${ }^{6,8-13}$ Neste trabalho, apresentamos 23 pacientes com 57 aneurismas intracranianos tratados cirurgicamente em um único estágio em nosso serviço hospitalar.

\section{Método}

Realizou-se um estudo retrospectivo, com base na análise dos prontuários hospitalares pertencentes a pacientes internados e em seguimento ambulatorial em nosso serviço hospitalar (Hospital Heliópolis), incluindo nesta pesquisa pacientes submetidos à clipagem de dois ou mais aneurismas cerebrais, em uma única cirurgia, no período de janeiro de 2007 a julho de 2012. Todas as cirurgias desta série foram realizadas/ tutoriadas pelos cirurgiões assistentes JCRJ, RO e MNS. Este estudo foi conduzido após aprovação no comitê de ética de nosso serviço hospitalar.

As seguintes informações foram coletadas dos prontuários hospitalares dos pacientes incluídos neste estudo: sexo e idade; graduação nas escalas de Hunt e Hess ${ }^{14}$ na admissão hospitalar; graduação na escala de Fisher et al. ${ }^{15}$ na admissão hospitalar; diagnóstico ou não de ruptura do aneurisma cerebral; localização e tamanho dos aneurismas cerebrais na angiografia cerebral pré-operatória; lateralidade da craniotomia utilizada; graduação na escala de Rankin seis meses após o tratamento cirúrgico.

\section{Técnica cirúrgica}

Todos os pacientes deste estudo foram submetidos a tomografia computadorizada de crânio e angiografia cerebral por subtração digital dos quatro vasos, assim como à discussão de caso por equipe constituída por 12 neurocirurgiões e um neurorradiologista, posteriormente se selecionando a modalidade de tratamento (cirúrgica, endovascular, combinada, ou conservadora).

Em nosso serviço hospitalar, o tratamento cirúrgico é o tratamento preconizado para aneurismas intracranianos múltiplos, e o objetivo é a clipagem de todos os aneurismas intracranianos diagnosticados por meio de uma craniotomia e em um único estágio. Todavia, esse objetivo eventualmente não é exequível e, com efeito, priorizam-se, primariamente, o acesso ipsilateral e a clipagem do aneurisma intracraniano roto ou de maior risco de ruptura e, secundariamente, $o$ acesso ipsilateral e contralateral (por meio da mesma craniotomia) ou bilateral (por meio de uma segunda craniotomia) para clipagem dos outros aneurismas intracranianos diagnosticados.

\section{Resultados}

Entre janeiro de 2009 e janeiro de 2011, 29 pacientes com 82 aneurismas intracranianos foram submetidos à clipagem de dois ou mais aneurismas cerebrais em uma única cirurgia. Vinte pacientes foram submetidos à clipagem de dois aneurismas; sete, à clipagem de três; e dois, à clipagem de quatro ou mais aneurismas, em uma única cirurgia. Cinco pacientes eram do sexo masculino e 24, do sexo feminino. A faixa etária esteve entre 40 e 66 anos, com média de 53 anos.

Vinte cinco pacientes apresentavam diagnóstico de hemorragia subaracnóidea; desses, à admissão, seis estavam graduados em I na escala de Hunt e Hess, cinco em II, 11 em III e três em IV. Da mesma forma, dois estavam graduados em II na escala de Fisher, 13 em III e 10 em IV. Os pacientes sem diagnóstico de ruptura de aneurisma cerebral, por convenção, foram classificados em 0 nas escalas de Hunt e Hess e I na escala de Fisher. Não obstante, dos 70 aneurismas cerebrais submetidos à clipagem, 25 foram considerados $\operatorname{rotos}^{13}$ e 57 , incidentais. Doze aneurismas incidentais foram tratados de forma conservadora (um desses, diagnosticado no paciente $n^{\circ} 3$, foi tratado de forma cirúrgica em um segundo estágio). 
Vinte e oito pacientes desta série foram submetidos à clipagem microcirúrgica de dois ou mais aneurismas intracranianos em um único estágio e por meio de uma única craniotomia. Um paciente ( $\left.n^{\circ} 25\right)$ foi submetido à clipagem microcirúrgica de dois aneurismas intracranianos em um único estágio por meio de duas craniotomias. Sessenta e sete dos aneurismas cerebrais clipados estavam localizados na circulação anterior e três, na circulação posterior, 26 no lado direito e 23 no lado esquerdo. Onze craniotomias à esquerda (oito pterionais, duas craniotomias fronto-orbitárias e uma frontotemporoparietal) foram realizadas para abordagem de 17 aneurismas cerebrais lateralizados à esquerda e cinco lateralizados à direita, assim como três aneurismas de complexo comunicante anterior. Dezoito craniotomias à direita (12 craniotomias pterionais, quatro fronto-orbitárias, uma frontotemporoparietal e uma frontal) foram realizadas para abordagem de 23 aneurismas cerebrais lateralizados à direita e seis lateralizados à esquerda, assim como 15 aneurismas de complexo comunicante anterior e um de artéria basilar (Tabela 1).

\begin{tabular}{|c|c|c|c|c|c|c|}
\hline \multirow{3}{*}{ Ruptura } & \multicolumn{5}{|c|}{$\begin{array}{c}\text { Tabela } 1 \text { - Características cirúrgicas de } 29 \text { pacientes submetidos a tratamento microcirúrgico } \\
\text { para aneurismas intracranianos múltiplos em um único estágio }\end{array}$} & \multirow{3}{*}{ Craniotomia } \\
\hline & \multirow[t]{2}{*}{ Paciente } & \multirow{2}{*}{$\begin{array}{l}\text { No de aneurismas } \\
\text { cerebrais clipados }\end{array}$} & \multicolumn{3}{|c|}{ Localização dos aneurismas cerebrais clipados } & \\
\hline & & & Direita & Central & Esquerda & \\
\hline \multirow[t]{4}{*}{ Não } & 01 & 2 & & & $\begin{array}{l}\mathrm{ACI}(\mathrm{Com}) \\
\mathrm{ACM}(\mathrm{M} 1)\end{array}$ & Esquerda \\
\hline & 02 & 2 & ACI (Com) & & ACI (Com) & Esquerda \\
\hline & 03 & 2 & $\mathrm{ACM}(\mathrm{M} 1)$ & & $\mathrm{ACI}(\mathrm{Oft})$ & Direita \\
\hline & 04 & 3 & ACI (Com) & AComA & $\mathrm{ACI}(\mathrm{Com})$ & Direita \\
\hline \multirow[t]{25}{*}{ Sim } & 05 & 3 & $\operatorname{ACM}(\mathrm{M} 1)$ & $\begin{array}{l}\operatorname{AComA} \\
\mathrm{AB}\end{array}$ & & Direita \\
\hline & 06 & 2 & $\underline{\operatorname{ACM}(M 1)}$ & & $\mathrm{ACI}(\mathrm{Com})$ & Direita \\
\hline & 07 & 2 & & AComA & ACI (Cor) & Esquerda \\
\hline & 08 & 2 & $\mathrm{ACM}(\mathrm{M} 1)$ & AComA & & Direita \\
\hline & 09 & 2 & $\mathrm{ACM}(\mathrm{M} 1)$ & AComA & & Direita \\
\hline & 10 & 3 & ACA (A2) & & $\frac{\operatorname{ACI}(\mathbf{O f t})}{\operatorname{ACM}(\mathrm{M} 1)}$ & Esquerda \\
\hline & 11 & 5 & $\begin{array}{l}\text { ACM (M1) } \\
\text { ACI (Com) }\end{array}$ & $\begin{array}{l}\operatorname{AComA} \\
\mathrm{AB}\end{array}$ & ACI (Com) & Direita \\
\hline & 12 & 4 & & $\begin{array}{l}\mathrm{AComA} \\
\mathrm{AB}\end{array}$ & $\frac{\text { ACI (Com) }}{\operatorname{ACM}(\mathrm{M} 1)}$ & Esquerda \\
\hline & 13 & 2 & ACI (Com) & & $\underline{\mathrm{ACI}(\mathrm{Com})}$ & Esquerda \\
\hline & 14 & 2 & ACI (Oft) & & $\underline{\mathrm{ACI}}(\mathrm{Oft})$ & Esquerda \\
\hline & 15 & 2 & $\underline{\mathrm{ACI}(\mathrm{Com})}$ & AComA & & Direita \\
\hline & 16 & 2 & $\frac{\operatorname{ACM}(M 1)}{\operatorname{ACM}(M 2)}$ & & & Direita \\
\hline & 17 & 2 & & & $\frac{\text { ACI (Com) }}{\text { ACI (Cor) }}$ & Esquerda \\
\hline & 18 & 2 & $\mathrm{ACM}(\mathrm{M} 1)$ & $\underline{\mathrm{ACom} A}$ & & Direita \\
\hline & 19 & 3 & ACI (Com) & $\underline{\mathrm{ACom} A}$ & $\mathrm{ACM}(\mathrm{M} 1)$ & Esquerda \\
\hline & 20 & 2 & $\underline{\operatorname{ACM}(\mathrm{M} 1)}$ & AComA & & Direita \\
\hline & 21 & 3 & $\begin{array}{l}\text { ACM (M2) } \\
\text { ACM (M1) }\end{array}$ & AComA & & Direita \\
\hline & 22 & 3 & $\mathrm{ACM}(\mathrm{M} 1)$ & $\underline{\mathrm{ACom} A}$ & ACI (Oft) & Direita \\
\hline & 23 & 2 & $\underline{\mathrm{ACI}(\mathrm{Com})}$ & AComA & & Direita \\
\hline & 24 & 2 & ACI (Cor) & & ACI (Cor) & Direita \\
\hline & 25 & 2 & $\mathrm{ACM}(\mathrm{M} 1)$ & & $\underline{\operatorname{ACM}(\mathrm{M} 1)}$ & Bilateral \\
\hline & 27 & 2 & & $\frac{\operatorname{AComA}}{A \operatorname{Com} A}$ & & Direita \\
\hline & 26 & 2 & ACI (Com) & $\underline{\mathrm{ACom} A}$ & & Direita \\
\hline & 28 & 2 & $\underline{\mathrm{ACA}}(\mathrm{A} 2)$ & & ACM (M2) & Esquerda \\
\hline & 29 & 3 & $\begin{array}{c}\mathrm{ACM}(\mathrm{M} 1) \\
\mathrm{ACI}(\mathrm{Cor})\end{array}$ & AComA & & Direita \\
\hline
\end{tabular}

AcomA: complexo artéria comunicante anterior; ACM: artéria cerebral média; ACI: artéria carótida interna; ACA: artéria cerebral anterior; AB: artéria basilar; Com: segmento comunicante da artéria carótida interna; Oft: segmento oftálmico da artéria carótida interna; Cor: segmento coróideo da artéria carótida interna. 
Dos 29 pacientes tratados cirurgicamente, 28 evoluíram, seis meses após a cirurgia, com 1-3 pts na graduação na escala de Rankin e somente 1 com 4-5 pts nessa mesma escala. Catorze pacientes apresentaram complicações relacionadas à hemorragia subaracnóidea ou ao tratamento cirúrgico: sete pacientes com hidrocefalia (quatro com hidrocefalia não shunt-dependente e três com hidrocefalia shunt-dependente), quatro com vasoespasmo clínico, três com infarto cerebral pós-operatório e um com ressangramento. Não houve óbitos nesta série (Tabela 2).

\begin{tabular}{|c|c|c|c|c|c|}
\hline \multicolumn{6}{|c|}{$\begin{array}{l}\text { Tabela } 2 \text { - Características clínicas e prognóstico clínico de } \\
29 \text { pacientes submetidos a tratamento microcirúrgico para } \\
\text { aneurismas intracranianos múltiplos em um único estágio }\end{array}$} \\
\hline Ruptura & Paciente & $\begin{array}{l}\text { Idade } \\
\text { e sexo }\end{array}$ & $\begin{array}{c}\text { Hunt- } \\
\text { Hess }\end{array}$ & Fisher & Rankin \\
\hline \multirow[t]{4}{*}{ Não } & 01 & $54 / \mathrm{F}$ & 0 & - & 1 \\
\hline & 02 & $50 / \mathrm{F}$ & 0 & - & 0 \\
\hline & 03 & $59 / \mathrm{F}$ & 0 & - & 0 \\
\hline & 04 & $46 / \mathrm{F}$ & 0 & - & 0 \\
\hline \multirow[t]{25}{*}{ Sim } & 05 & $49 / \mathrm{F}$ & 2 & 4 & 1 \\
\hline & 06 & $50 / \mathrm{F}$ & 1 & 2 & 0 \\
\hline & 07 & $60 / \mathrm{F}$ & 3 & 4 & 0 \\
\hline & 08 & $49 / \mathrm{F}$ & 2 & 3 & 1 \\
\hline & 09 & $49 / \mathrm{F}$ & 3 & 4 & 1 \\
\hline & 10 & $42 / \mathrm{F}$ & 3 & 3 & 3 \\
\hline & 11 & $46 / \mathrm{M}$ & 3 & 4 & 2 \\
\hline & 12 & $53 / \mathrm{F}$ & 3 & 2 & 1 \\
\hline & 13 & $40 / \mathrm{F}$ & 1 & 4 & 1 \\
\hline & 14 & $66 / F$ & 3 & 3 & 2 \\
\hline & 15 & $53 / \mathrm{F}$ & 3 & 3 & 1 \\
\hline & 16 & $62 / \mathrm{F}$ & 1 & 4 & 2 \\
\hline & 17 & $53 / \mathrm{F}$ & 3 & 4 & 1 \\
\hline & 18 & $42 / \mathrm{M}$ & 4 & 4 & 1 \\
\hline & 19 & $43 / \mathrm{F}$ & 1 & 3 & 0 \\
\hline & 20 & $65 / \mathrm{M}$ & 1 & 1 & 0 \\
\hline & 21 & $52 / \mathrm{M}$ & 4 & 4 & 5 \\
\hline & 22 & $46 \mathrm{M}$ & 4 & 3 & 2 \\
\hline & 23 & $61 / F$ & 1 & 4 & 0 \\
\hline & 24 & $28 / \mathrm{F}$ & 2 & 3 & 0 \\
\hline & 25 & $47 / \mathrm{F}$ & 3 & 3 & 2 \\
\hline & 27 & $40 / \mathrm{F}$ & 2 & 3 & 1 \\
\hline & 26 & $54 / \mathrm{F}$ & 3 & 3 & 1 \\
\hline & 28 & $41 / F$ & 2 & 3 & 1 \\
\hline & 29 & $44 / \mathrm{F}$ & 3 & 3 & 2 \\
\hline
\end{tabular}

\section{Discussão}

Bigelow, ${ }^{16}$ em 1955, publicou a primeira revisão da literatura sobre aneurismas intracranianos múltiplos, identificando em uma série 2.237 casos de aneurismas intracranianos, uma frequência de $10 \%$ de aneurismas intracranianos múltiplos. Atualmente, essa frequência é superior à descrita por Bigelow e calculada em torno de $15 \%-45 \%$, provavelmente devido a um aumento na utilização, na qualidade e na quantidade de vasos estudados nas angiografias cerebrais por subtração digital e às políticas de encaminhamento. ${ }^{17}$

$\mathrm{Na}$ atualidade, as duas modalidades de tratamento mais utilizadas e eficazes no tratamento de aneurismas intracranianos são a clipagem microcirúrgica e a embolização com coil. A decisão para se utilizar o tratamento cirúrgico ou endovascular é baseada na morfologia do aneurisma, na idade e no status clínico do paciente, ${ }^{2}$ assim como na técnica e experiência do neurocirurgião ou do neurorradiologista. Conforme a literatura, o tratamento cirúrgico apresenta uma frequência de obliteração completa superior e um risco de recanalização inferior em relação ao tratamento endovascular e, com efeito, permanece como a modalidade terapêutica mais adequada principalmente em pacientes com idade inferior a 50 anos e com status clínico razoável, ${ }^{2}$ todavia, não há um consenso sobre esse assunto, tendo em vista que há aumento do número de estudos indicando morbimortalidade inferior e eficácia similar (principalmente com o advento de novas tecnologias) do tratamento endovascular em comparação com o tratamento microcirúrgico.

Em nossa casuística, 24 dos 29 casos eram do sexo feminino (da mesma forma, na literatura, há predomínio do sexo feminino nos casos de aneurismas intracranianos múltiplos), ${ }^{4,7,17}$ a faixa etária esteve entre 40 e 60 anos (na literatura, é o grupo etário com maior incidência de hemorragia subaracnóidea aneurismática $)^{4,7}$ e, assim como em todos os estudos revisados, ${ }^{7,8,10,11,15-20}$ a ruptura aneurismática foi a forma de apresentação mais comum (25 casos dos 29 pacientes), tornando a identificação pré-operatória do aneurisma roto condição sine qua non para a decisão e planejamento da estratégia terapêutica. A identificação do sítio de ruptura aneurismática foi realizada por meio de um algoritmo elaborado por Nehls et al., ${ }^{18}$ em 1985, com base em informações obtidas da tomografia de crânio, da angiografia cerebral e do exame clínico de pacientes com aneurismas intracranianos múltiplos. Dos 25 casos com diagnóstico de ruptura aneurismática, 14 pacientes foram admitidos com comprometimento moderado a grave do status neurológico (III e IV na escala de Hunt e Hess) ${ }^{14}$ e 14 evoluíram com complicações importantes associadas à hemorragia subaracnóidea. Não houve óbito nesta série, e apenas dois pacientes (caso $n^{\circ} 10$ e 21), após seis meses do tratamento cirúrgico, foram pontuados com três ou mais pontos na escala de Rankin; ambos apresentavam um comprometimento de moderado a grave do status neurológico no pré-operatório e evoluíram com 
complicações importantes decorrentes da hemorragia subaracnóidea. Em consonância com a literatura, a hemorragia subaracnóidea e o status clínico e neurológico pré-operatório são os principais fatores determinantes do prognóstico do tratamento cirúrgico de aneurismas intracranianos múltiplos, somados à idade do paciente e à presença de aneurismas intracranianos gigantes, complexos ou localizados na circulação posterior. ${ }^{16}$

Dos 29 pacientes submetidos ao tratamento cirúrgico para aneurismas intracranianos múltiplos em um único estágio, 20 foram submetidos à clipagem de dois aneurismas intracranianos e nove à clipagem de mais de dois aneurismas intracranianos (somente dois foram submetidos à clipagem de mais de três aneurismas intracranianos). Os sítios de localização mais frequentes foram a artéria carótida interna (24 aneurismas - 14 localizados no segmento comunicante) e o complexo comunicante anterior (18 aneurismas). Da mesma forma, a artéria carótida interna e o complexo comunicante anterior foram os sítios de ruptura mais comuns (oito aneurismas rotos localizados na artéria carótida interna - seis de segmento comunicante - e 11 aneurismas rotos no complexo comunicante anterior). Considerando somente as lesões duplas, as combinações mais frequentes foram: um aneurisma localizado na artéria carótida interna, somado a um segundo aneurisma no complexo comunicante anterior (cinco combinações; desses, quatro aneurismas no segmento comunicante e um no segmento oftálmico da artéria carótida interna); e um aneurisma localizado na artéria carótida interna, somado a um segundo na artéria carótida interna (cinco combinações; desses, quatro combinações no segmento comunicante da artéria carótida interna implicando a disposição em espelho).

Conforme a literatura, há três formas de abordagem microcirúrgica dos aneurismas intracranianos múltiplos em um único estágio: o acesso unilateral, o contralateral e o bilateral. ${ }^{15,20} \mathrm{O}$ acesso unilateral é, mais frequentemente, utilizado para clipagem de aneurismas intracranianos localizados central e ipsilateralmente na circulação anterior e na parte superior da circulação posterior; o acesso contralateral (por meio de uma única craniotomia) e o acesso bilateral (por meio de duas craniotomias) são, mais frequentemente, utilizados para aneurismas localizados contralateralmente. A localização unilateral de múltiplos aneurismas intracranianos na circulação anterior facilita a decisão pelo tratamento cirúrgico de todos os aneurismas intracranianos e um único estágio (aneurismas intracranianos múltiplos localizados na circulação anterior contralateral ou na circulação posterior)..$^{15,21}$ Em nosso estudo, 28 pacientes foram submetidos ao tratamento cirúrgico de aneurismas intracranianos múltiplos por meio de uma única craniotomia, e somente um paciente foi submetido a duas craniotomias e, portanto, ao acesso bilateral. Utilizou-se a via contralateral para a clipagem microcirúrgica de 10 aneurismas incidentais (nove de artéria carótida interna e um de artéria cerebral anterior distal) localizados no hemisfério contralateral à craniotomia (duas craniotomias fronto-orbitárias ou oito pterionais) em 10 pacientes. $\mathrm{O}$ acesso contralateral é uma técnica de microcirurgia sistematizada por Oliveira et al..$^{20} \mathrm{e}$ Vajda et al. ${ }^{22}$ Um único acesso para clipagem de aneurismas localizados ipsi e contralateralmente, se viável, é preconizado em todos os casos de múltiplos aneurismas intracranianos em nosso serviço hospitalar, por proporcionar o tratamento definitivo em um único estágio e com uma única craniotomia, consequentemente diminuindo o tempo de internação e não adicionando risco de complicações inerentes a uma segunda craniotomia.

Doze aneurismas intracranianos incidentais referentes a 11 pacientes foram tratados de forma conservadora; desses, quatro eram de segmento cavernoso da artéria carótida interna; um era de segmento oftálmico da artéria carótida interna (paciente $\mathrm{n}^{\circ} 3$ ), com tamanho de $4 \mathrm{~mm}$ e com sinais de trombose intraluminal e calcificação parietal; quatro eram aneurismas intracranianos com tamanho inferior a $2 \mathrm{~mm}$ ( 1 de PICA no segmento cortical no paciente $\mathrm{n}^{\circ} 10$, dois de artéria cerebral média nos pacientes $n^{\circ} 11$ e $n^{\circ} 25$, dois de artéria carótida interna no segmento comunicante nos pacientes $\mathrm{n}^{\circ} 15$ e $\mathrm{n}^{\circ} 29$, e um de artéria cerebral anterior no segmento A3 no paciente $n^{\circ} 21$ ) e estavam localizados distante ou contralateralmente ao acesso; e um aneurisma de artéria carótida interna no segmento comunicante, com tamanho de $4 \mathrm{~mm}$, foi tratado cirurgicamente em um segundo estágio e uma segunda internação hospitalar, por causa de dificuldades para a clipagem microcirúrgica no primeiro estágio. Conforme Rinne et al. ${ }^{21}$ apesar de uma política cirúrgica ativa, até um terço dos aneurismas intracranianos diagnosticados pode permanecer sem tratamento, a maioria deles em pacientes com aneurismas intracranianos múltiplos. Comprometimento grave a severo do status clínico, idade avançada e relutância do paciente, assim como aneurismas intracranianos considerados inviáveis ao tratamento cirúrgico, seriam as principais razões para um tratamento conservador. ${ }^{23}$

\section{Conclusão}

Em nossa casuística, 29 pacientes com aneurismas intracranianos múltiplos foram submetidos à clipagem microcirúrgica de dois ou mais aneurismas intracranianos (totalizando 71 aneurismas intracranianos submetidos à clipagem microcirúrgica), incluindo 10 
localizados na circulação anterior contralateralmente ao acesso e três localizados na circulação posterior, em um único estágio e por meio de uma (em 28 pacientes) ou duas (somente em um paciente) craniotomias. Não houve complicações importantes adicionadas pelo tratamento cirúrgico, e apenas dois dos 29 pacientes desta série evoluíram com comprometimento moderado a grave do status funcional seis meses após a cirurgia, em consequência de complicações relacionadas à hemorragia subaracnóidea. Preconizamos abordagem microneurocirúrgica para a maioria dos casos de aneurismas intracranianos múltiplos, buscando a clipagem microcirúrgica de todos os aneurismas intracranianos, se viável, em um único estágio e por meio de uma única craniotomia.

\section{Conflito de interesses}

Os autores declaram não haver conflito de interesses.

\section{Referências}

1. Ellamushi HE, Grieve JP, Jäger HR, Kitchen ND. Risk factors for the formation of multiple intracranial aneurysms. J Neurosurg. 2001;94(5):728-32.

2. Rinne J, Hernesniemi J, Puranen M, Saari T. Multiple intracranial aneurysms in a defined population: prospective angiographic and clinical study. Neurosurgery. 1994;35(5):803-8.

3. Ostergaard JR, Høg E. Incidence of multiple intracranial aneurysms. Influence of arterial hypertension and gender. J Neurosurg. 1985;63(1):49-55.

4. Inagawa T. Surgical treatment of multiple intracranial aneurysms. Acta Neurochir (Wien). 1991;108(1-2):22-9.

5. Mizoi K, Suzuki J, Yoshimoto T. Surgical treatment of multiple aneurysms. Review of experience with 372 cases. Acta Neurochir (Wien). 1989;96(1-2):8-14.

6. Report of World Federation of Neurological Surgeons Committee on a Universal Subarachnoid Hemorrhage Grading Scale. J Neurosurg. 1988;68(6):985-6.

7. Vajda J. Multiple intracranial aneurysms: a high risk condition. Acta Neurochir (Wien). 1992;118(1-2):59-75.

8. Juvela S. Risk factors for multiple intracranial aneurysms. Stroke. 2000;31(2):392-7.

9. Drake CG, Girvin JP. The surgical treatment of subarachnoid hemorrhage with multiple aneurysms. In: Morley TP, editor. Current controversies in neurosurgery. Philadelphia: Saunders; 1976. p. 274-8.
10. Lawton MT, Quinones-Hinojosa A, Sanai N, Malek JY, Dowd CF. Combined microsurgical and endovascular management of complex intracranial aneurysms. Neurosurgery. 2003;52(2):263-74.

11. Mckissock W, Richardson A, Walsh L, Owen E. multiple intracranial aneurysms. Lancet. 1964;1(7334):623-6.

12. Molyneux AJ, Kerr RS, Birks J, Ramzi N, Yarnold J, Sneade $M$, et al. Risk of recurrent subarachnoid haemorrhage, death, or dependence and standardised mortality ratios after clipping or coiling of an intracranial aneurysm in the International Subarachnoid Aneurysm Trial (ISAT): long-term follow-up. Lancet Neurol. 2009;8(5):427-33.

13. Qureshi Al, Suarez JI, Parekh PD, Sung G, Geocadin R, Bhardwaj $A$, et al. Risk factors for multiple intracranial aneurysms. Neurosurgery. 1998;43(1):22-6.

14. Hunt WE, Hess RM. Surgical risk as related to time of intervention in the repair of intracranial aneurysms. J Neurosurg. 1968;28(1):14-20.

15. Fisher CM, Kistler JP, Davis JM. Relation of cerebral vasospasm to subarachnoid hemorrhage visualized by computerized tomographic scanning. Neurosurgery. 1980;6(1):1-9.

16. Bigelow $\mathrm{NH}$. Multiple intracranial arterial aneurysms; an analysis of their significance. AMA Arch Neurol Psychiatry. 1955;73(1):76-99.

17. Orz Y, Osawa M, Tanaka Y, Kyoshima K, Kobayashi S. Surgical outcome for multiple intracranial aneurysms. Acta Neurochir (Wien). 1996;138(4):411-7.

18. Nehls DG, Flom RA, Carter LP, Spetzler RF. Multiple intracranial aneurysms: determining the site of rupture. $J$ Neurosurg. 1985;63(3):342-8.

19. Vajda J, Juhász J, Orosz E, Pásztor E, Tóth S, Horváth M. Surgical treatment of multiple intracranial aneurysms. Acta Neurochir (Wien). 1986;82(1-2):14-23.

20. Oliveira E, Ribas GC, Yamashita M, Lourenzi M, Marino R Jr. Abordagem fronto-temporo-esfenoidal para a clipagem de aneurismas múltiplos localizados ipsi e contralateralmente. In: $16^{\circ}$ Congresso Brasileiro de Neurocirurgia, Rio de Janeiro, 1986.

21. Rinne J, Hernesniemi J, Niskanen M, Vapalahti M. Management outcome for multiple intracranial aneurysms. Neurosurgery. 1995;36(1):31-7.

22. Vajda J, Juhász J, Pásztor E, Nyáry I. Contralateral approach to bilateral and ophthalmic aneurysms. Neurosurgery. 1988;22(4):662-8

23. Bohman LE, Winn HR, LeRoux PD. Surgical decision making for treatment of intracranial aneurysms. In: Youmans JR. Neurological surgery. 6th ed. Philadelphia: Saunders; 2011. p. $3766-8$

\section{Endereço para correspondência:}

Pablo Rodrigues de Carvalho

Hospital Heliópolis

Av. Senador Roberto Simonsen, 403,

ap. 51-B, Santo Antônio

09530-401 - São Caetano do Sul, SP, Brasil

Telefone: (11) 98460-5085

E-mail: ber2511@yahoo.com.br 